\title{
Commentary: Current National Institutes of Health funding for cardiac surgery-Is the glass half full or half empty?
}

\author{
Harold L. Lazar, MD \\ From the Boston University School of Medicine, Boston, Mass. \\ Disclosures: Author has nothing to disclose with regard to commercial support. \\ Received for publication Aug 19, 2019; revisions received Aug 19, 2019; accepted for publication Aug 20, 2019; \\ available ahead of print Oct 11, 2019. \\ Address for reprints: Harold L. Lazar, MD, Boston University School of Medicine, 80 East Concord St, Boston, \\ MA 02118 (E-mail: harold.1.lazar@gmail.com). \\ J Thorac Cardiovasc Surg 2020;159:2336-7 \\ $0022-5223 / \$ 36.00$ \\ Copyright $(c) 2019$ by The American Association for Thoracic Surgery \\ https://doi.org/10.1016/j.jtcvs.2019.08.125
}

The volume of basic science and investigator-initiated research published by cardiac surgeons has declined. Today, the vast majority of manuscripts submitted to cardiovascular journals by cardiac surgeons consist of case reports, observational studies, single-center case series, and metaanalyses. There are several explanations for this decrease in basic science research among cardiac surgeons:

1. More time is spent dealing with clinical responsibilities that are necessary for treating high-risk patients undergoing more complex procedures in an era of open public reporting.

2. There is decreased institutional support for initiating research activities even among "university-affiliated" hospitals.

3. There is a belief among hospital administrators that time spent conducting basic science research results in less clinical productivity, and ultimately, less clinical revenue.

4. There is currently a shortage of cardiac surgical "mentors" who are well versed in performing high-quality basic science research.

In this edition of the Journal, Narahari and colleagues ${ }^{1}$ sought to determine the current level of cardiac surgery research activity by determining trends in National Institutes of Health (NIH) funding for cardiac surgeons in 141 $\mathrm{NIH}$-funded institutions. In their central message, the authors concluded that they had "shown that cardiac surgeon scientists excel at obtaining NIH grants." However, a closer look at the data indicates that this might not be totally accurate.

The majority of NIH funding to cardiac surgeons was concentrated in the top quartile of the 141 institutions that received NIH funding and was strongly correlated with an institution's overall NIH funding rank. Of a total of $818 \mathrm{ac}-$ ademic cardiac surgeons, only $17.6 \%$ received some type of $\mathrm{NIH}$ funding. Although the number of new NIH grants to

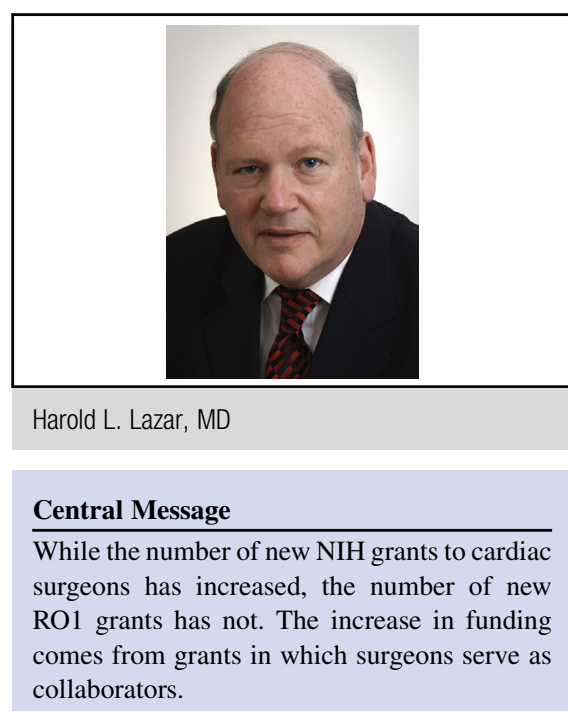

See Article page 2326.

cardiac surgeons and the maintenance of active grants has increased over time, the number of new RO1 grants has not increased. This comes at a time when $\mathrm{NIH}$ funding has increased over the past 25 years. The authors found that the overall increase in NIH grants to cardiac surgeons was driven primarily by "P" and " $U$ " grants, which are reflective of collaborative projects, involving large research teams and multiple mentors rather than a single, primary PI. The authors note that as the difficulty in obtaining RO1 grants increase, cardiac surgeons have "opted for a team based approach to perform scientific research." But what, exactly, does this mean-are cardiac surgeons actively involved in the planning, analysis of data, and writing the manuscripts and proposals, or are they merely providing tissue and specimens for basic scientists to analyze? The authors postulate that these top-quartile institutions are more conducive to cardiac surgeons receiving NIH support due to the infrastructure and available mentorship at these institutions. However, is it merely that these institutions are more likely to have ongoing funded projects to which cardiac surgeons can more easily participate. The authors have not clearly defined the role of cardiac surgeons in these "collaborative grants."

Another area that the authors have not addressed is the relevance of current $\mathrm{NIH}$ grants to the practice of clinical 
cardiac surgery. In previous years, NIH-supported research led directly to innovations in myocardial protection with cardioplegia, the treatment of acute respiratory distress syndrome and cardiomyopathies with mechanical support, the identification and mechanisms for systemic inflammatory response syndrome and its management with complement suppression and inhibition, methods for improved immunosuppression following heart and lung transplantation, and neuroprotection during deep hypothermic circulatory arrest. All these research efforts have led directly to decreased morbidity and mortality in cardiac surgery patients. Today's funded NIH research is more oriented toward identifying "mechanisms" by which clinical interventions can be instituted to improve patient outcomes. These include gene therapy, angiogenesis with cardiac stem cells, and genetically engineered organ transplantation. These interventions may take longer periods of time to demonstrate clinical efficacy and may never become part of clinical practice protocols. Other NIH-funded translation studies such as the reasons for hospital readmissions after cardiac surgery, remodeling following mitral valve surgery, and the role of 3-dimensional echo in surgical valve analysis may merely serve to support the conclusions of multiple single-center studies that have already been published.

While the authors have provided us with data to show that the cardiac surgeon scientist receiving NIH funding is not extinct, it would appear that this species can only survive in certain academic environments. Although the authors have made NIH funding a surrogate for cardiac surgery research activity, high-quality research can still be conducted and receive research funding in institutions without a history of NIH funding, provided that cardiac surgeons have access to a strong database and the support of a statistician. If the cardiac surgeon scientist species is to survive, these investigator-initiated, non-NIH funded studies will be crucial. Only then can we determine whether if in cardiac surgery research, the glass is half full or half empty.

\section{Reference}

1. Narahari AK, Cook IO, Mehaffey JH, Chandrabhatla AS, Hawkins RB, Tyerman Z, et al. Comprehensive National Institutes of Health funding analysis of academic cardiac surgeons. J Thorac Cardiovasc Surg. 2020;159:2326-35.e4. 